For personal use only. Not to be reproduced without permission of the publisher (editorial@gabi-journal.net).

\title{
Why bioequivalence and uncon- ditional interchangeability of generic drugs are not the same
}

\author{
Professor Teun van Gelder, MD, PhD
}

\section{The patents for a number of cornerstone immunosuppres- sive drugs used in the field of solid organ transplantation} have expired. Generic formulations are now available and several professional societies and individual physicians have given their opinions on the issue of generics substitution. In this issue of GaBl Journal, several hot topics related to implementation of generic immunosuppressive drugs in clinical practice were discussed in different perspectives.

Keywords: Bioequivalence, generics substitution, immunosuppression, transplantation

\begin{abstract}
Introduction
This paper focuses on the immunosuppressive drugs used in the field of solid organ transplantation. Within the treatment of organ transplant recipients, roughly three eras can be distinguished. Before 1980, treatment consisted of high dose corticosteroids and azathioprine, often combined with anti-thymocyte globulin induction therapy. With this treatment the majority of renal transplant patients developed one or more acute rejection episodes, and the one-year graft survival was close to $50 \%$. In the early 80 s, ciclosporin was introduced, and this revolutionized pharmacotherapy. The incidence of acute rejection not only decreased, rejections were also less severe, and graft survival at one year increased to $80 \%$. For nonrenal organs the impact was even more dramatic. Results of liver, lung and heart transplantation were very poor prior to the introduction of ciclosporin. In the years after 1982 many centres were able to successfully start liver, heart or lung transplant programmes. The third era started in 1995, when tacrolimus was introduced as an alternative for ciclosporin. Both drugs are calcineurin inhibitors, but their side
\end{abstract}

effect profiles differ and with tacrolimus lower incidences of acute rejection were achieved (although one-year graft survival was similar). Mycophenolate mofetil also became available in the mid 1990s, and was shown to reduce the incidence of acute rejection by $50 \%$, compared to azathioprine-based immunosuppressive therapy [1].

Combined treatment with tacrolimus and mycophenolate mofetil has become the most frequently used regimen to prevent acute rejection after renal transplantation. For both drugs the patent has expired, both in the US and in most European countries. Generic formulations have reached the market, but there is debate regarding the pros and cons of generics substitution.

In this paper I will not discuss pharmaceutical quality. As a medical doctor I assume that drugs that have received marketing authorization meet the rigorous quality standards for product identity, strength, and purity. National and international regulators check whether or not drug products are manufactured under the strict standards required for good manufacturing practices.

Evidence that generics substitution is either safe or risky is lacking. The studies that have attempted to relate substitution to clinical outcome are underpowered. While there are case histories published concerning this, the evidential value of these cases is small at best. As a result there are many expert opinions on this subject. I have personally contributed to the ongoing debate, with my personal opinions, as both the secretary of the Dutch Society for Transplantation and as the Chair of an advisory committee of the European Society for Organ Transplantation [2]. For the record, this editorial reflects my personal opinion and the contents cannot be linked to either of the two societies.

\section{The patient}

There are many stakeholders involved in this issue, and as always, money plays an important role. One viewpoint that is often forgotten is the opinion of the patient. For patients, the function of their transplanted organ is of utmost importance. For liver, heart and lung transplants these grafts are life saving. For renal transplant patients the grafts offer much better quality of life compared to dialysis. Many patients were on waiting lists for substantial periods of time prior to transplantation, and they are very much aware of their poor prospects in case of graft loss. Patients who have been treated with the originator drug for a considerable length of time, with good experience, feel uncertain about any proposed switch to another formulation, even with their doctor's consent. Surveys show that a large percentage of renal transplant patients believe that generics are not equivalent and many patients are uncertain whether generics have the same quality as do innovator medicines [3]. When generics substitution is intentionally or unintentionally forced upon such patients this may adversely affect adherence to medication, potentially influencing clinical outcome. There definitely should be more information for patients on this topic, in language that they can understand.

The confusion that patients experience due to substitution from one formulation 
to the other [4] is also important. In many countries purchasing policies of either the pharmacist or the health insurance company will lead to dispensing of numerous generic formulations from various sources over time. Not all of my patients will be able to figure out that boxes that look so different and are labelled with different names in fact contain the same drug [5]. The resulting confusion can and will certainly lead to mistakes. In view of the narrow therapeutic index of transplant medications such mistakes may have serious consequences. Following the first substitution from an innovator drug to a generic formulation there should be no subsequent generic-generic substitutions. Prescription of a so-called branded generic drug may help to avoid such subsequent substitutions. Dispensing of immunosuppressive drugs only through one or more designated pharmacies may provide a way to achieve better control over what product is handed to the patient. And it would certainly help if registration authorities would demand that the shape and colour of the tablets and capsules of generic formulations are the same or at least similar to the innovator medicines they can replace. This would help patients to correctly identify their medication, and could reduce confusion and mistakes.

\section{Registration}

Immunosuppressive drugs are considered narrow therapeutic index drugs. For such drugs relatively small changes in exposure may lead to differences in clinical outcome. For many years it was felt that the limits for the $90 \%$ confidence intervals $\left(90 \% \mathrm{CI}\right.$ ) of the $\mathrm{C}_{\max }$ and AUC ratios, between the two preparations should be stricter than the range of $0.80-1.25$.

The European Medicines Agency currently recommends that the acceptance interval be tightened for products for which there is a risk of clinically relevant differences in efficacy or safety between two products even when the conventional criteria for assuming bioequivalence are met. For such products the acceptance interval for concluding bioequivalence should be narrowed to $0.9-1.11 \%$. The US Food and Drug Administration has not changed its policy, and in the US the wider range $(80-125 \%)$ is still applied even for narrow therapeutic index drugs [6].

What is not required for registration is the need to demonstrate bioequivalence with other generic formulations of the same drug. This is surprising, since the acceptance criteria for generic formulations have been chosen to minimize the risks associated with substitution of one formulation with another. Substitutions of one generic formulation with another generic formulation are likely to happen when pharmacists may assume that the prescriber has agreed to use any generic formulation once a first substitution has taken place. The dispensing pharmacist may therefore not inform the prescriber of any such subsequent changes in what the patient actually takes. I understand that for most drugs the generic formulations have ratios for $\mathrm{C}_{\max }$ and $\mathrm{AUC}$ that are close to the middle of the range of 0.8-1.25. For such drugs the changes in exposure following substitution are assumed to be small, but this is not necessarily true for all drugs. I would expect that registration authorities would build in safeguards to prevent this from happening, even if this would occur in less than 5\% of all drugs [6]. In my view the requirement to not only demonstrate bioequivalence with the innovator drug but also with other generic formulations would increase the safety of generics substitution. If such testing were required a company that wanted to register a new generic formulation of a drug for which there were already five other formulations on the market, the registration hurdle would become higher. But do we really need a sixth generic version? In this issue Dr Marc Maliepaard, working for the Dutch Medicines Evaluation Board, discusses an alternative view of the potential changes in exposure following substitution from one generic drug to another.

Another issue in the registration process which is often debated is whether healthy volunteers are the appropriate study population. Some argue that for immunosuppressive drugs bioequivalence studies should be done in transplanted patients. I personally do not believe that patients are the preferred population. The higher degree of intra-subject variability in patients would complicate the demonstration of bioequivalence. Studies in patients would have to include substantially more subjects to bring the 90\% confidence intervals for the ratios within the bioequivalence limits. In some cases, to fulfill the perceived need for bioequivalence studies in actual patient populations, generics manufacturers have sponsored studies in transplanted patients [7] even when such studies were not required by the registration authorities. The main goal of such studies was to convince transplant physicians that the generic product was also bioequivalent with the innovator drug in the target patient groups.

It has been pointed out that although the innovator and generic formulation contain the same amount of the active substance, differences in excipients may alter the disposition of co-administered drugs. An example of this phenomenon is the study by Kovarik et al. [8], where sirolimus pharmacokinetics were different in the presence of generic versus innovator ciclosporin.

\section{Therapeutic equivalence}

Therapeutic equivalence is assumed on the basis of demonstrated comparative bioavailability ('bioequivalence'). There is no regulatory requirement to perform clinical trials comparing clinical outcomes in patients treated with the innovator versus the generic formulation. I believe that it is unlikely that a difference in drug exposure of less than $10 \%$ will result in a difference in clinical outcome, and I would not personally be interested in participating in a trial to test this hypothesis. More importantly, if such a non-inferiority trial would be done it would not reflect daily practice. In the trial setting, patients would all be supplied with a single study drug, either innovator or generic, for the duration of the study. Throughout the study patients in both arms of the study would remain on the same formulation. In daily practice, however, subsequent and often uncontrolled substitutions from one generic formulation to another take place. As already mentioned above, these substitutions may result in deviations from the therapeutic target concentrations or compliance, and more importantly will lead to confusion and mistakes that could affect outcomes. Head-to-head comparisons between an innovator drug and a specific generic formulation do not reflect clinical practice, and cannot provide evidence for the safety of repeated generics substitution [6].

\section{Implementation}

In The Netherlands, generics substitution is the standard of practice for drugs such as proton pump inhibitors, anti-hypertensive drugs and for statins. Increasing numbers of physicians use an electronic prescription system, within which the default is the generic drug name. In my practice transplanted patients are still treated with inno- 
vator immunosuppressive drugs. On my prescriptions for immunosuppressive drugs I add the trade [brand] name, and the words 'medical necessity'. This tells the pharmacist that I do not want a generic substitution. My main motivation is the fear for subsequent repetitive substitutions. As described above such subsequent substitutions are likely to happen without my knowledge. As a result, I would not be alerted to check drug exposure, and even if I do check drug concentrations I cannot check whether or not the patient is taking the drugs correctly. And again, patients may make mistakes. If there would be a guarantee that following a first substitution from innovator immunosuppressive drug to a specific generic drug under controlled conditions that there would be no further substitutions, this would certainly change my position on the use of generic anti-rejection drugs.

\section{Conclusion}

This issue of GaBI Journal offers you different perspectives on the generic substitution of immunosuppressive drugs. Regulators, insurers, pharmacists, physicians and patients are all stakeholders in this controversial area. Hopefully, you will find useful new perspectives and opinions in this issue. However, do not expect to find much evidence that substitution is always safe.

Competing interests: Professor Teun van Gelder has received honoraria, research grants or lecture fees from Astellas, Pfizer, Roche, Sandoz and Wyeth; and is a member of the Dutch Novartis Transplant Advisory Board.

Provenance and peer review: Commissioned; externally peer reviewed.

\section{References}

1. Halloran PF. Immunosuppressive drugs for kidney transplantation. N Engl J Med. 2004 Dec 23;351(26): 2715-29.

2. van Gelder T. European Society for Organ Transplantation Advisory Committee recommendations on generic substitution of immunosuppressive drugs. Transpl Int. 2011 Dec;24(12):1135-41.
3. Al Ameri MN, Whittaker C, Tucker A, Yaqoob M, Johnston A. A survey to determine the views of renal transplant patients on generic substitution in the UK. Transpl Int. 2011 Aug;24(8):770-9.

4. Greene JA. The substance of the brand. Lancet. 2011 Jul 9;378(9786):120-1.

5. Greene JA, Kesselheim AS. Why do the same drugs look different? Pills, trade dress, and public health. N Engl J Med. 2011 Jul 7;365(1):83-9.

6. van Gelder T, Gabardi S. Methods, strengths, weaknesses, and limitations of bioequivalence tests with special regard to immunosuppressive drugs. Transpl Int. 2013 Feb 27. doi:10.1111/tri. 12074 .

7. Alloway RR, Sadaka B, Trofe-Clark J, Wiland A, Bloom RD. A randomized pharmacokinetic study of generic tacrolimus versus reference tacrolimus in kidney transplant recipients. Am J Transplant. 2012 Oct;12(10):2825-31.

8. Kovarik JM, Noe A, Wang Y, Mueller I, DeNucci G, Schmouder RL. Differentiation of innovator versus generic cyclosporine via a drug interaction on sirolimus. Eur J Clin Pharmacol. 2006 May;62(5): 361-6.

DOI: $10.5639 /$ gabij.2013.0202.020

Copyright (c) 2013 Pro Pharma Communications International 